\title{
Regression or Pseudo-Inverse - Which Method Should be Preferred When Developing Inverse Linear ECG-Lead Transformations?
}

\author{
Daniel Guldenring ${ }^{1}$, Ali Rababah ${ }^{2}$, Dewar D Finlay ${ }^{2}$, Raymond R Bond ${ }^{2}$, Alan Kennedy ${ }^{2}$, \\ Michael Jennings ${ }^{2}$, Khaled Rjoob ${ }^{2}$, James McLaughlin ${ }^{2}$ \\ ${ }^{1}$ HS Kempten, Kempten, Germany \\ ${ }^{2}$ Ulster University, Belfast, United Kingdom
}

\begin{abstract}
Linear ECG-lead transformations estimate or derive unrecorded target leads by applying a number of recorded basis leads to a so-called linear ECG-lead transformation matrix. The inverse transform of such a linear ECG-lead transformation performs a transformation in the opposite direction (from the target leads to the basis leads). The pseudo-inverse of a given transformation matrix can be used to perform such an inverse transformation. Linear regression based inverse transformation matrices are, provided that sufficient training data for their development is available, an alternative to pseudo-inverse matrices. The aim of this research was to compare the estimation performance of pseudo-inverse and linear regression based inverse transformations. This comparison was performed for two example inverse transformations. The performance of the different transformations was assessed using root-meansquared-error (RMSE) values between the QRS-T complexes of recorded and derived leads. Typical mean RMSE values associated with the regression based approach were found to be approximately two thirds to half of the mean RMSE values achieved by the approach based upon the pseudo-inverse. Provided that sufficient data are available, linear regression should be used for the development of inverse ECG-lead transformation matrices.
\end{abstract}

\section{Introduction}

Linear ECG-lead transformations estimate or derive unrecorded target leads by applying a number of recorded basis leads to a so-called linear ECG-lead transformation matrix $[1,2]$. The inverse transform of such a linear ECG-lead transformation performs a transformation in the opposite direction (from the target leads to the basis leads). The pseudo-inverse [3] of a given transformation matrix can be used to perform such an inverse transformation. A well-known example of such a pseudoinverse based inverse ECG-lead transformation matrix is the so-called inverse Dower transformation [4]. Linear regression based inverse transformation matrices are, provided that sufficient training data for their development is available, an alternative to pseudo-inverse based inverse ECG-lead transformation matrices. However, a comparison between the estimation performance of pseudo-inverse and linear regression based inverse ECG-lead transformations has, to the best of our knowledge, not previously been reported in the literature. The aim of our research was to compare the estimation performance of pseudo-inverse and linear regression based inverse ECG-lead transformations. This was performed for two example inverse linear ECG-lead transformations. More precisely, we compared the performance of inverse ECG-lead transformations for transformations from the Frank VCG to the standard 12lead ECG and for transformations in the opposite direction.

\section{Material and methods}

\subsection{Study population}

We base our research on a study population of 726 subjects. The study population is composed of 229 normal subjects, 265 subjects with myocardial infarction and 232 subjects with left ventricular hypertrophy. The study population was randomly partitioned into a training dataset (DTrain) and a test dataset (DTest). Table 1 details the composition of DTrain and DTest.

Table 1. Composition of train data (DTrain) and test data (DTest).

\begin{tabular}{lcccc}
\hline & Normal & MI & LVH & Total \\
\hline DTrain & 172 & 199 & 174 & 545 \\
DTest & 57 & 66 & 58 & 181 \\
\hline
\end{tabular}

Notes. Normal, Subjects with no abnormalities in their ECGs; MI, Subjects with myocardial infarction; LVH, Subjects with left ventricular hypertrophy.

\subsection{BSPM data}

One body surface potential map (BSPM) was recorded for each of the 726 subjects in the study population. Each BSPM used in this research contains electrocardiographic data of 120 BSPM leads. A representative average 
QRS-T complex was calculated for each of the 120 BSPM leads. Three of the 120 leads were recorded from electrodes placed on the right and left wrist and the left ankle (VR, VL and VF respectively). Electrodes situated at 81 anterior and 36 posterior locations were used to record 117 thoracic leads. All thoracic leads were recorded with reference to the Wilson central terminal (WCT). A comprehensive description of the BSPM data and the recording procedure can be found in $[5,6]$. A Laplacian 3D interpolation procedure [7] was applied to the 117 thoracic BSPM leads. This was performed to obtain body surface potentials at the locations of the 352 Dalhousie torso [8] nodes.

\subsection{Frank VCG data}

One Frank VCG [9] was extracted from each of the 726 BSPMs. First, body surface potentials at the A, C, E, F, H, I and M electrode locations of the Frank lead system were extracted from the interpolated BSPM data. Required body surface potentials from body locations that were not a direct subset of the 352 nodes that are used by the Dalhousie torso were obtained using linear interpolation [10]. Second, the body surface potentials at the Frank electrode locations were used to derive the Frank VCG using (1).

$$
V C G=[X, Y, Z]=\left[\varphi_{A}, \ldots, \varphi_{M}\right] \cdot A^{T} .
$$

Where $\varphi_{A}, \varphi_{C}, \varphi_{E}, \varphi_{F}, \varphi_{H}, \varphi_{I}$, and $\varphi_{M}$ are $n \times 1$ vectors that contain $n$ sample values of potentials at the Frank electrode locations A to $\mathrm{M}$ respectively, $[\cdot]^{T}$ refers to the transpose of a matrix, $n$ denotes the number of samples in the average QRS-T complex, $\boldsymbol{A}$ is a $3 \times 7$ matrix of published coefficients [11] that allow for a derivation of the Frank VCG using the potentials $\boldsymbol{\varphi}_{A}$ to $\boldsymbol{\varphi}_{\boldsymbol{M}}$, and $\boldsymbol{V C G}$ is a $n \times 3$ matrix containing $n$ sample values of the Frank VCG, the $n \times 1$ vectors $\boldsymbol{X}, \boldsymbol{Y}$ and $\boldsymbol{Z}$ contain $n$ sample values of the three Frank leads $\mathrm{X}, \mathrm{Y}$ and $\mathrm{Z}$ respectively.

\subsection{Standard 12-lead ECG data}

One standard 12-lead ECG [12] was extracted from each of the 726 BSPMs. First, body surface potentials recorded at the wrists and ankles were used to calculate the limb leads of the standard 12-lead ECG and the potential at the WCT. Second, the body surface potentials at the electrode locations associated with the precordial leads were extracted from the interpolated BSPM data. Required body surface potentials from locations that were not a direct subset of the 352 Dalhousie torso nodes were obtained using linear interpolation [10]. Third, average QRS-T complexes of the precordial leads were calculated in reference to the WCT using the body surface potentials obtained from the locations of the precordial electrodes.

\subsection{Linear regression based ECG lead transformation matrices}

Two linear ECG lead transformation matrices were developed using multivariate linear regression [13] and the data in DTrain. More precisely a transformation matrix that allows for the transformation from the standard 12-lead ECG to the Frank VCG and a transformation matrix that allows for the transformation from the Frank VCG to the standard 12-lead VCG were developed. This was performed using the approach in (2a) and (2b).

$$
\begin{aligned}
A 12 L^{r e g} & =\left(V C G^{T} \cdot V C G\right)^{-1} \cdot V C G^{T} \cdot S 12 L \\
A V C G^{r e g} & =\left(S 12 L^{T} \cdot S 12 L\right)^{-1} \cdot S 12 L^{T} \cdot V C G
\end{aligned}
$$

Where $[\cdot]^{T}$ and $[\cdot]^{-1}$ denote the transpose and the inverse of a matrix respectively, $\boldsymbol{A 1 2} \boldsymbol{L}^{\text {reg }}$ refers to a $3 \times 12$ matrix of transformation coefficients that allows for the transformation of the Frank VCG into the standard 12-lead ECG, $\boldsymbol{A V} \boldsymbol{C} \boldsymbol{G}^{\text {reg }}$ refers to a $12 \times 3$ matrix of transformation coefficients that allows for the transformation of the standard 12-lead ECG into the Frank VCG, $\boldsymbol{V} \boldsymbol{C G}$ refers to a $n \times 3$ matrix that contains $n$ sample values of the $\mathrm{X}, \mathrm{Y}$ and $\mathrm{Z}$ leads of the Frank VCG, $S 12 L$ refers to a $n \times 12$ matrix that contains $n$ sample values of the eight independent leads I, II and V1 to V6 of the standard 12-lead ECG and $n$ denotes the total number of QRS-T samples for each ECG lead in DTrain.

\subsection{Pseudo invers of the linear ECG lead transformation matrices}

One inverse lead transformation for each of the two linear regression based ECG lead transformation matrices was developed. This was achieved by calculation the pseudo-inverse of the two different lead transformation matrices. More precisely, the pseudo-inverse of the regression based VCG to 12-lead transformation matrix $\boldsymbol{A 1 2} \boldsymbol{L}^{\text {reg }}$ was determined using (3a) and the pseudoinverse of the regression based 12-lead ECG to VCG transformation matrix $\boldsymbol{A V} \boldsymbol{C} \boldsymbol{G}^{\text {reg }}$ was determined using (3b).

$$
\begin{aligned}
& A V C G^{\text {pin }}=\left(A 12 L^{r e g}\right)^{T} \cdot\left(A 12 L^{r e g} \cdot\left(A 12 L^{r e g}\right)^{T}\right)^{-1} \\
& A 12 L^{p i n}=\left(\left(A V C G^{r e g}\right)^{T} \cdot A V C G^{r e g}\right)^{-1} \cdot\left(A V C G^{r e g}\right)^{T} .
\end{aligned}
$$

Where $\boldsymbol{A 1 2 L ^ { r e g }}, \boldsymbol{A V C G} \boldsymbol{G}^{\text {reg }},[\cdot]^{T}$ and $[\cdot]^{-1}$ are as defined in (2a) and (2b), $\boldsymbol{A} \boldsymbol{V} \boldsymbol{C} \boldsymbol{G}^{\boldsymbol{p} \text { in }}$ denotes a $12 \times 3$ pseudo-inverse based matrix of transformation coefficients that allows for the transformation of the standard 12-lead ECG into the Frank VCG and $\boldsymbol{A 1 2}^{\text {pin }}$ refers to a $3 \times 12$ matrix of transformation coefficients that allows for the transformation of the Frank VCG into the 
standard 12-lead ECG.

\subsection{Derivation of the target leads}

Regression and pseudo-inverse based transformation matrices were used to derive their respective target lead sets. More precisely, the matrices $\boldsymbol{A} \boldsymbol{V} \boldsymbol{C} \boldsymbol{G}^{\boldsymbol{r e g}}$ and $\boldsymbol{A} \boldsymbol{V} \boldsymbol{C} \boldsymbol{G}^{\boldsymbol{p i n}}$ were used to derive Frank VCGs using standard 12-lead ECG data for each of the 181 subjects in DTest using (4a) and (4b).

$d V C G^{r e g}=A V C G^{r e g} \cdot S 12 L$.

$d V C G^{p i n}=A V C G^{p i n} \cdot S 12 L$.

Where $\boldsymbol{A} \boldsymbol{V} \boldsymbol{C} \boldsymbol{G}^{\boldsymbol{r e g}}$ is as defined in (2b) and $\boldsymbol{A} \boldsymbol{V} \boldsymbol{C} \boldsymbol{G}^{\boldsymbol{p} \boldsymbol{i n}}$ is as defined in (3a), $\boldsymbol{S 1 2} \boldsymbol{L}$ is a $n \times 8$ matrix that contains the $n$ sample values of the QRS-T complex for the eight independent leads of the standard 12-lead ECG of one subject in DTest, $\boldsymbol{d} \boldsymbol{V} \boldsymbol{C G}_{\boldsymbol{i}}^{\boldsymbol{r e g}}$ and $\boldsymbol{d} \boldsymbol{V} \boldsymbol{C} \boldsymbol{G}_{\boldsymbol{i}}^{\boldsymbol{p i n}}$ are $n \times 3$ matrices that contain the derived leads of the Frank VCG computed from regression and pseudo-inverse based transformation matrices respectively.

In addition, the matrices $\boldsymbol{A} 12 \boldsymbol{L}^{\boldsymbol{r e g}}$ and $\boldsymbol{A 1 2} \boldsymbol{L}^{\boldsymbol{p i n}}$ were used to derive the standard 12-lead ECG from Frank VCG data for each of the 181 subjects in DTest using (5a) and (5b).

$d 12 L^{r e g}=A 12 L^{r e g} \cdot V C G$.
$d 12 L^{p i n}=A 12 L^{p i n} \cdot V C G$.

Where $\boldsymbol{A 1 2} \boldsymbol{L}^{\boldsymbol{r e g}}$ is as defined in (2a) and $\boldsymbol{A 1 2} \boldsymbol{L}^{\boldsymbol{p i n}}$ is as defined in (3b), $\boldsymbol{V} \boldsymbol{C G}$ is a $n \times 3$ matrix that contains the $n$ sample values of the QRS-T complex for the three leads of the Frank VCG of one subject in DTest, $\boldsymbol{d 1 2} \boldsymbol{L}^{\text {reg }}$ and $\boldsymbol{d 1 2}^{\text {pin }}$ are $n \times 8$ matrices that contain the derived leads of the eight independent leads of the standard 12-lead ECG computed from regression and pseudo-inverse based transformation matrices respectively.

\subsection{Performance assessment}

The performance of the different ECG lead transformation matrices was quantified using the data in DTest. First, root mean square error (RMSE) values were calculated between the recorded and the derived ECG leads. This was performed for each of the four transformation matrices and for each of the 181 subjects in DTest. Second, the mean [95\% confidence intervals (CI)] and the standard deviation of the 181 different RMSE values associated with each target lead and transformation method were determined. Third, the statistical significance of the differences in the mean RMSE values between regression and pseudo-inverse based transformation methods was assessed (two-tailed, paired t-test, significance level alpha $=0.05)$.

\section{Results}

The findings of the performance assessment associated with the inverse transforms from the 12-lead ECG to the Frank VCG and the inverse transformations in the opposite direction are provided in Table 1 and Table 2 respectively.

Table 1. Mean and standard deviation of the root mean square error values calculated between derived and recorded leads of the Frank VCG.

\begin{tabular}{ccccc}
\hline $\begin{array}{c}\text { Derived } \\
\text { lead }\end{array}$ & $\begin{array}{c}\text { Transformation } \\
\text { method }\end{array}$ & $\begin{array}{c}\text { Mean } \\
{[95 \% \mathrm{CI}]^{\mathrm{a}}}\end{array}$ & $\begin{array}{c}\text { Standard } \\
\text { deviation }^{\mathrm{a}}\end{array}$ & $\begin{array}{c}\mathrm{p}- \\
\text { value }^{\mathrm{b}}\end{array}$ \\
\hline \multirow{2}{*}{$\mathrm{X}$} & Regression & $30.1[26.7 ; 33.4]$ & 22.6 & $<0.001$ \\
& Pseudo-inverse & $63.2[56.5 ; 69.8]$ & 45.4 & \\
\hline \multirow{2}{*}{$\mathrm{Y}$} & Regression & $30.3[26.2 ; 34.5]$ & 28.3 & $<0.001$ \\
& Pseudo-inverse & $48.0[43.9 ; 52.0]$ & 27.6 & \\
\hline \multirow{2}{*}{$\mathrm{Z}$} & Regression & $47.0[42.6 ; 51.3]$ & 29.6 & $<0.001$ \\
& Pseudo-inverse & $64.0[59.0 ; 68.9]$ & 33.4 & \\
\hline
\end{tabular}

amean and standard deviation of the RMSE values are provided in $\mu \mathrm{V} ;{ }^{b}$ p-value obtained from a two-tailed, paired t-test for the statistical significance of the difference in the mean RMSE value associated with regression and pseudo-inverse based transformation matrices.

Table 2. Mean and standard deviation of the root mean square error values calculated between derived and recorded leads of the standard 12-lead ECG.

\begin{tabular}{|c|c|c|c|c|}
\hline $\begin{array}{c}\text { Derived } \\
\text { lead }\end{array}$ & $\begin{array}{c}\text { Transformation } \\
\text { method }\end{array}$ & $\begin{array}{c}\text { Mean } \\
{[95 \% \mathrm{CI}]}\end{array}$ & $\begin{array}{l}\text { Standard } \\
\text { deviation }\end{array}$ & $\begin{array}{c}\mathrm{p}- \\
\text { value }\end{array}$ \\
\hline \multirow{2}{*}{ I } & Regression & $65.0[58.7 ; 71.3]$ & 43.0 & \multirow{2}{*}{$<0.001$} \\
\hline & Pseudo-inverse & $156.8[145.8 ; 167.8]$ & 75.1 & \\
\hline \multirow{2}{*}{ II } & Regression & $37.5[31.6 ; 43.4]$ & 40.1 & \multirow{2}{*}{$<0.001$} \\
\hline & Pseudo-inverse & $60.2[52.9 ; 67.5]$ & 49.8 & \\
\hline \multirow{2}{*}{ V1 } & Regression & $85.5[77.8 ; 93.3]$ & 52.9 & \multirow{2}{*}{$<0.001$} \\
\hline & Pseudo-inverse & $194.7[181.6 ; 207.8]$ & 89.2 & \\
\hline \multirow{2}{*}{$\mathrm{V} 2$} & Regression & $182.8[167.7 ; 197.8]$ & 102.7 & \multirow{2}{*}{$<0.001$} \\
\hline & Pseudo-inverse & $526.0[491.6 ; 560.4]$ & 234.5 & \\
\hline \multirow{2}{*}{ V3 } & Regression & $113.7[103.9 ; 123.5]$ & 66.9 & \multirow{2}{*}{$<0.001$} \\
\hline & Pseudo-inverse & $209.5[191.0 ; 228.1]$ & 126.4 & \\
\hline \multirow{2}{*}{ V4 } & Regression & $150.6[132.2 ; 169.0]$ & 125.7 & \multirow{2}{*}{$<0.001$} \\
\hline & Pseudo-inverse & $394.0[365.3 ; 422.8]$ & 195.8 & \\
\hline \multirow{2}{*}{ V5 } & Regression & $98.9[90.3 ; 107.5]$ & 58.7 & \multirow{2}{*}{$<0.001$} \\
\hline & Pseudo-inverse & $286.1[261.1 ; 311.1]$ & 170.4 & \\
\hline \multirow{2}{*}{ V6 } & Regression & $58.7[52.2 ; 65.3]$ & 44.6 & \multirow{2}{*}{$<0.001$} \\
\hline & Pseudo-inverse & $105.7[96.4 ; 114.9]$ & 63.0 & \\
\hline
\end{tabular}

${ }^{a}$ mean and standard deviation of the RMSE values are provided in $\mu \mathrm{V} ;{ }^{b}$-value obtained from a two-tailed, paired t-test for the statistical significance of the difference in the mean RMSE value associated with regression and pseudo-inverse based transformation matrices. 


\section{Discussion and conclusion}

This paper reported on the estimation performance of different inverse ECG-lead transformations. One pseudoinverse and one linear regression based transformation matrix was developed for each of the investigated inverse ECG-lead transformations.

Our findings show differences in the estimation performance of linear regression and pseudo-inverse based invers ECG-lead transformations. The observed differences were found to be in favor for the regression based approach. Typical mean RMSE values associated with the regression based approach were found to be approximately two thirds to half of the mean RMSE values achieved by the approach based upon the pseudoinverse. For example, the RMSE values (mean; [95\% confidence interval]) when estimating Frank VCG lead X from the 12-lead ECG using the linear regression and the pseudo-inverse approach were found to be $30 \mu \mathrm{V} ;[27 \mu \mathrm{V}$; $33 \mu \mathrm{V}]$ and $63 \mu \mathrm{V} ;[57 \mu \mathrm{V} ; 70 \mu \mathrm{V}]$ respectively.

Linear regression is based upon the minimization of the squared errors between derived and recorded target leads on the training dataset. Regression based transformation matrices represent therefore the best (in the least squares sense) linear transformation of the recoded basis leads into the estimated target leads. Regression based inverse ECG-lead transformations will therefore lead to estimates, that are at least as good or better than what can be achieved by linear transformations that are developed using the pseudoinverse. This, however, is provided that the regression based inverse ECG-lead transformations are developed using a sufficiently sized training dataset that is reprehensive of the study population. It is evident that an unrepresentative and or small training dataset might lead to regression based inverse ECG-lead transformations that are associated with higher estimation errors when compared to what is achieved by the pseudo-inverse of a linear ECG-lead transformation that has been developed using an representative and sufficiently sized training dataset.

A limitation of this research is that it has not been assessed or specified what constitutes a sufficiently sized and representative dataset. However, based upon the findings of this research we recommend the following procedure when developing inverse ECG-lead transformations. Provided sufficient data are available, one should develop regression based inverse ECG-lead transformations and compare their estimation performance against their pseudo-inverse counterparts. This approach allows for the identification and utilization of the best performing inverse ECG-lead transformation for a given application.

\section{References}

[1] Guldenring D, Finlay DD, Strauss DG, et al. Transformation of the Mason-Likar 12-lead electrocardiogram to the Frank vectorcardiogram. Conf Proc IEEE Eng Med Biol Soc. 2012;2012:677-680.

[2] Guldenring D, Finlay DD, Bond RR, et al. The derivation of the spatial QRS-T angle and the spatial ventricular gradient using the Mason-Likar 12-lead electrocardiogram. $J$ Electrocardiol. 2015;48(6):1045-1052.

[3] Golan JS, The Moore-Penrose Pseudoinverse. In: Foundations of Linear Algebra. Dordrecht: Springer; 1995. pp 198-203.

[4] Edenbrandt L, Pahlm O. Vectorcardiogram synthesized from a 12-lead ECG: superiority of the inverse Dower matrix. J Electrocardiol 1988; 21(4):361-367.

[5] Kornreich F, Montague T, Rautaharju P. Identification of first acute $\mathrm{Q}$ wave and non-Q wave myocardial infarction by multivariate analysis of body surface potential maps. Circulation 1991; 84(6): 2442-2453.

[6] Montague TJ, Smith ER, Cameron DA, Rautaharju PM, Klassen GA, Felmington CS, et al. Isointegral analysis of body surface maps: surface distribution and temporal variability in normal subjects. Circulation 1981; 63(5): 1166-1172.

[7] Oostendorp TF, van Oosterom A, Huiskamp G. Interpolation on a triangulated 3D surface. J Comput Phys 1989; 80(2):331343.

[8] Horáček BM. Numerical Model of an Inhomogeneous Human Torso. Adv Cardiol 1974; 10:51-57.

[9] Frank E. An accurate, clinically practical system for spatial vectorcardiography. Circulation 1956; 13(5):737-749.

[10] Schijvenaars BJA, Kors JA, van Herpen G, Kornreich F, van Bemmel JH. Interpolation of body surface potential maps. J Electrocardiol 1995; 28 Suppl 1: 104-109.

[11] Macfarlane PW. Lead systems. In: Macfarlane PW, van Oosterom A, Pahlm O, Kligfield P, Janse M, Camm J, editors. Comprehensive Electrocardiology. 2nd ed. United Kingdom. London: Springer; 2011; p. 375-426.

[12] Kligfield P, Gettes LS, Bailey JJ, Childers R, Deal BJ, Hancock EW, et al. Recommendations for the Standardization and Interpretation of the Electrocardiogram: Part I: The Electrocardiogram and Its Technology: A Scientific Statement From the American Heart Association Electrocardiography and Arrhythmias Committee, Council on Clinical Cardiology; the American College of Cardiology Foundation; and the Heart Rhythm Society Endorsed by the International Society for Computerized Electrocardiology. Circulation 2007; 115(10):1306-1324.

[13] Izenman AJ. Multivariate Regression. In: Modern Multivariate Statistical Techniques. New York: Springer; 2013; p.159-194.

Address for correspondence.

Daniel Guldenring

Room T117, Faculty of Electrical Engineering, HS Kempten, Bahnhofstraße 61, 87435 Kempten, Germany

daniel.gueldenring@hs-kempten.de 